\title{
CAMBIOS EN LA DISTRIBUCIÓN Y ABUNDANCIA DE LA ICTIOFAUNA DE AGUAS SOMERAS EN SAN BARTOLO (LIMA, PERÚ) DESPUÉS DEL ENSO 1997-98
}

\section{DISTRIBUTION CHANGES AND ABUNDANCE OF SHALLOW WATER'S ICTIOFAUNA IN SAN BARTOLO (LIMA, PERÚ) AFTER THE ENSO 1997-1998}

\author{
Antonio Gárate $^{1}$ y Aldo Pacheco ${ }^{2}$
}

\begin{abstract}
Resumen
El evento El Niño 1997-98, trajo consigo la tropicalización de las aguas en la bahía sur de San Bartolo, lo que produjo cambios en la ictiofauna del lugar. Con el objetivo de evaluar y reportar la variabilidad en un periodo posterior a la perturbación, se realizaron observaciones mediante buceo, durante los meses de Febrero - Mayo de 1999 y Enero - Julio del 2000. Los resultados de este estudio muestran que, especies son propias de la Provincia Panameña incursionaron en la bahía, estas a su vez dejaron de observarse conforme paso el tiempo de estudio. Otras especies usuales para la Provincia Peruano - Chilena, en general, no sufrieron cambios en su distribución. Como conclusión general, el presente estudio muestra que, durante el post ENSO las especies generan diferentes respuestas, algunas provenientes de la provincia Panameña son capaces de expandir sus límites usuales de distribución hacia la bahía, pero desapareciendo conforme pasa el tiempo, interpretándose esto como el proceso de resiliencia, posterior a la perturbación. Otras presentan una gran capacidad de aclimatación ante el ENSO.
\end{abstract}

Palabras clave: Peces, distribución, abundancia, ENSO, Provincia biogeográfica.

\begin{abstract}
The tropicalization of shallow waters of Bahía Sur at San Bartolo beach by El Niño Event was shown by changes in the distribution of fishes. In order to evaluate and report the variability during a period after perturbation, diving observations were made between February - May 1999, and January - July 2000. The results of this study show that species of the Panamanian Province, which invaded the bay expanding their latitudinal distribution during El Niño disappeared progressively through the study period, while other local residents of the Peruvian - Chilean province did not change their typical distribution. As a general conclusion, it can be stated that, during the post ENSO, species generate different responses: some of the species from the Panamanian Province are able to expand their natural distribution limits, and then progressively disappear, while other species show a great capacity of acclimation under this natural disturbance. Key words: Fishes, distribution, abundance, ENSO, Biogeographic province.
\end{abstract}

\section{Introducción}

El evento El Niño Oscilación Sur (ENSO) de 1997-98 se caracterizó por generar cambios en los factores abióticos del ecosistema marino peruano. Particularmente importante fue el aumento de la temperatura superficial del mar, registrándose anomalías de hasta $6^{\circ} \mathrm{C}$, y la disminución de la disponibilidad de nutrientes, asociadas a la expansión de Aguas Ecuatoriales frente a todo el litoral de la Corriente de Humboldt de aguas frías. Además, se produjo una baja en la productividad del mar, por la declinación de los procesos de afloramiento (Espino, 1999). Esta variación en las condiciones ambientales, también generó múltiples respuestas en las diferentes poblaciones de animales y algas. Las especies experimentaron aumento en sus densidades poblaciones, altas mortalidades, emigraciones e inmigraciones, cambios en la actividad reproductora, y cambios en los rangos de distribución, latitudinal, longitudinal y en profundidad (Espino, 1999; Mendo \& Wolff 2003; Jahncke et al., 2004).
Para el grupo particular de los peces se ha estudiado diferentes aspectos relacionados con la variabilidad inducida por el ENSO, como la dinámica poblacional de especies pelágicas por ejemplo; anchoveta y sardina, especies demersales como la merluza (Espino et al., 1985; Samame et al., 1985), también, aspectos tróficos para especies de aguas someras en la Bahía de Ancón (Hoyos et al., 1985), y para especies sujetas a explotación pesquera (Velez \& Zeballos, 1985; Sánchez de Benites et al., 1985). En bahías someras, se genera una dinámica particular, puesto que estos lugares sirven de áreas de reclutamiento para las especies de peces ya que encuentran condiciones favorables para su desarrollo (Hooker, 1998). Estos sistemas también han sufrido el efecto modificador del ENSO en donde se han reportado cambios en la distribución y abundancia de peces (Hoyos et al., 1985).

Durante el ENSO algunas especies de peces amplían sus rangos de distribución hasta latitudes donde habitualmente no se encuentran, generaron 


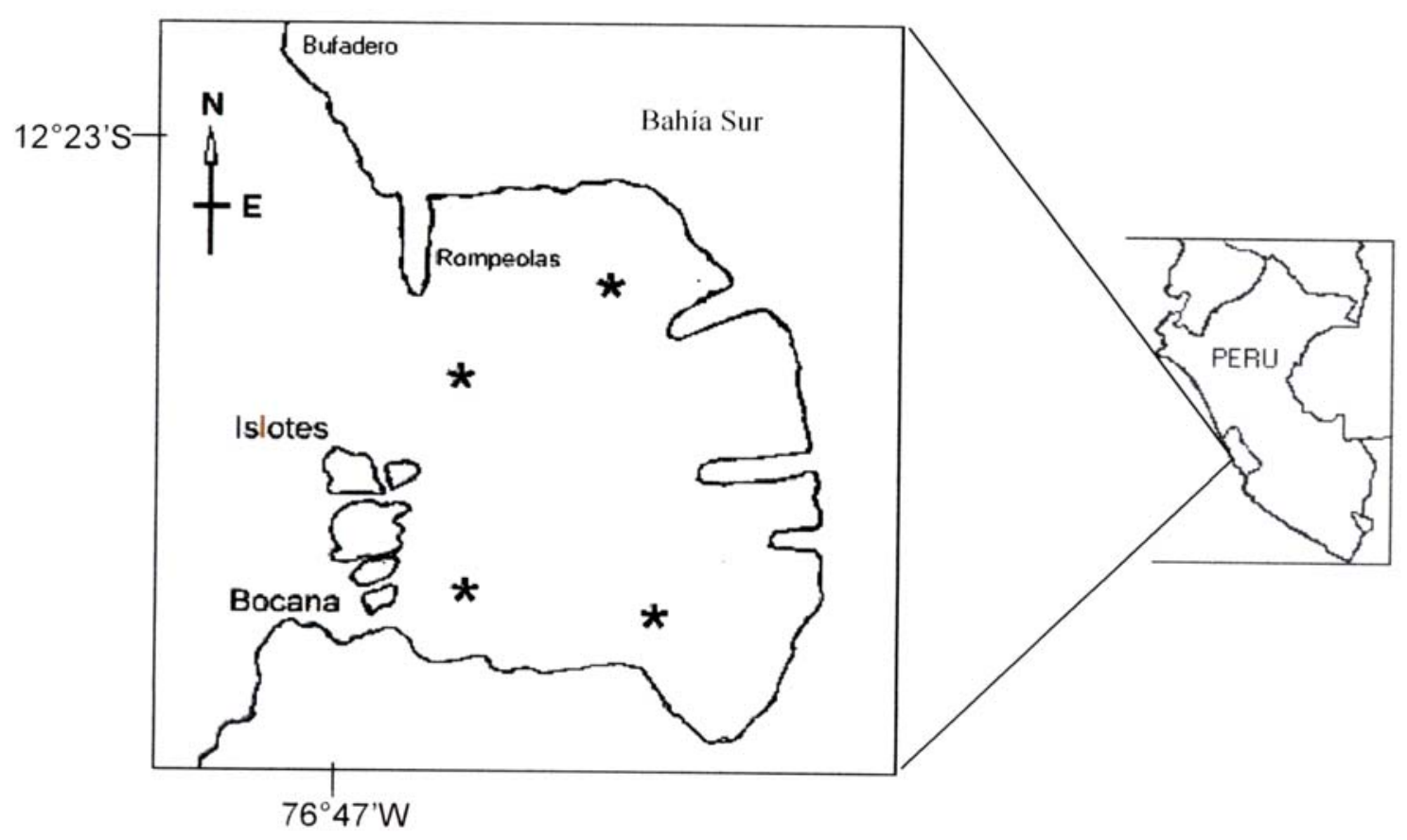

Figura 1. Área de estudio bahía sur balneario San Bartolo y la ubicación de los puntos de observación.

pesquerías alternativas, lo que se considera como un impacto positivo del evento en la actividad humana. La ampliación de las distribuciones estuvo representada por especies que vienen de la zona norte y de la zona oceánica (Espino, 1999).

El balneario de San Bartolo es un pequeño sistema conformado por tres bahías, dos de las cuales son semiprotegidas del oleaje, en donde se practicó buceo, después del ENSO 1997-98, con lo que se pudo registrar la ictiofauna del lugar. El objetivo de este estudio es presentar información sobre la riqueza de especies, abundancia y cambios en la distribución de especies de peces en la zona durante el periodo post ENSO 1997-98. Esta información resulta importante puesto que contribuye al ordenamiento y manejo de aquellas pesquerías ocasionales que se generan durante estos eventos, además permite conocer la respuesta de la ictiofauna ante esta perturbación natural.

\section{Materiales y Métodos \\ Área de estudio}

La bahía Sur se encuentra ubicada a $50 \mathrm{~km}$ al sur de Lima (12 $23^{\prime}$ S, $\left.76^{\circ} 47^{\prime} \mathrm{W}\right)$ (Figura 1), en el distrito de San Bartolo. El litoral está conformado por tres pequeñas bahías ubicadas consecutivamente. Bahía Sur se encuentra al centro de las 3 bahías y está delimitada por una saliente rocosa denominada
Bufadero y se prolonga hasta el sector denominado La Bocana, constituido por una saliente de roca y una serie de cinco islotes. Esta bahía es la más pequeña y está protegida del oleaje por una serie de pequeños islotes al sur oeste y por una isla de mayor tamaño hacia el oeste. La boca de la bahía es ancha y la playa ubicada hacia el interior, está conformada por bolones sobre sedimentos blandos. El promedio de profundidad es de $2.5 \mathrm{~m}$ y la máxima profundidad se ubica hacia el centro de la boca a $4 \mathrm{~m}$. El fondo está constituido por bolones, piedras grandes y paredones, alternados por parches de arena y grava. En los lados de la bahía el fondo está conformado por macizos de roca de color blanco. Hacia el norte se ubica la Bahía Norte y hacia el sur limita con la Bahía Curayacu. Usualmente, el lugar presenta condiciones calmas, lo que permite practicar buceo.

\section{Método de muestreo}

Utilizando equipo de buceo SCUBA, se realizaron observaciones quincenales entre los meses de Febrero - Mayo de 1999 y entre Enero - Julio del 2000, para lo cual se ubicaron cuatro puntos fijos de observación, señalados con una pequeña boya sujeta a un lastre (ver Figura 1). Dado que la mayoría de los peces presentan una gran movilidad, en cada punto de observación el buzo se situó en un lugar fijo y se observó dentro del campo visual generado por el ángulo de 180 grados frente al buzo. Se registraron las especies de peces que 
se encontraban en el área de observación durante 15 minutos. Una vez terminado el tiempo de registro, se realizaron observaciones alrededor del área entre las grietas y piedras que eventualmente, pudieron estar ocupadas por otros peces. La identificación taxonómica de las especies se corroboró con los trabajos de Humann (1993) y Chirichigno (1998).

Para el registro de la abundancia se generó una escala de aproximación, con los siguientes términos: (1) muy abundante; más de 20 individuos en el campo visual, (2) abundante; entre 10 y 20 individuos, (3) regular; entre 10 y 5 individuos, (4) escasa; entre 5 y 1 y (5) rara; 1 individuo. Se realizó un registro continuo de la abundancia, sin embargo debido a la variabilidad en presencia y abundancia temporal de las especies, sólo se consideró como dato, el último registro de abundancia de la especie. Adicionalmente se registró la temperatura superficial del mar durante el período de estudio.

\section{Resultados}

Se realizaron un total de ocho inmersiones para el año 1999 y doce para el 2000 lo que significó en total de 20 horas de observaciones. Se identificaron un total de 30 especies de peces pertenecientes a 22 familias, de las cuales 16 están descritas para la Provincia biogeográfica Panameña y 14 están descritas para la Provincia biogeográfica Peruano - Chilena (Tabla 1). La riqueza de las especies varió conforme fue avanzando el tiempo, encontrándose que, las especies cuya distribución corresponde a la Provincia Panameña dejan de presentarse conforme pasa la perturbación. En cambio la riqueza de las especies de la Provincia Peruano - Chilena mostró menos variación, manteniéndose de manera regular durante el periodo de observación (Figura 2). Las especies muy abundantes fueron: Cheilodactylus variegatus, Mugil cephalus y Sphyraena ensis. Abundantes fueron: Scarthictis gigas, Chaetodon humeralis, Halichoeres diispilus, Anisotremus scapularis y Labrisomus phillipii. Las de abundancia regular fueron: Hypsoblennius sordidus, Anisotremus interruptus, Chromis crusma, Nexilosus latifrons, Strongylura exilis, Doidixodon laevifrons, Oplegnathus insignis. Escasas; Abudefduf troschellii, Calamus brachypomus, Scomberomorus sierra. Finalmente de abundancia rara: Johnrandalia nigrirostris, Bodianus diplotaenia, Stegastes flavigatus, Mycteroperca xenarcha, Ephinephelus labriformis, Balistes polylepis, Seriola rivoliana, Fistularia corneta, Pseudopeneus grandisquamis, Nicholsina denticulada, Scorpaena histrio, Sphoeroides lobatus (Figura 3). La temperatura superficial en el periodo comprendido entre Febrero - Mayo de 1999 fue de $20.5^{\circ} \mathrm{C}$ y para el período Enero - Julio del 2000 fue de $17.5^{\circ} \mathrm{C}$ en promedio.

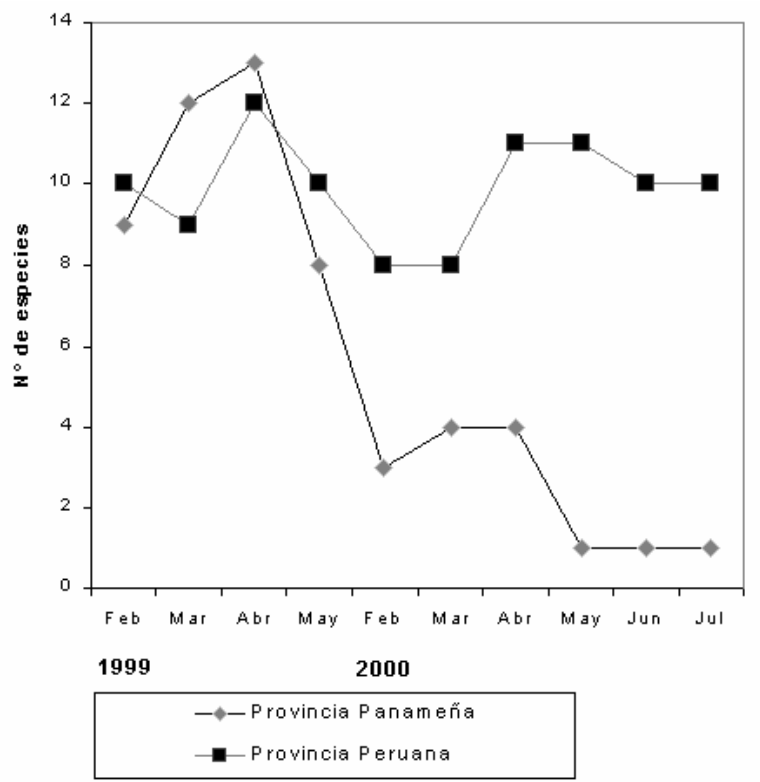

Figura 2. Variación de la riqueza de especies de peces durante el periodo de estudio.

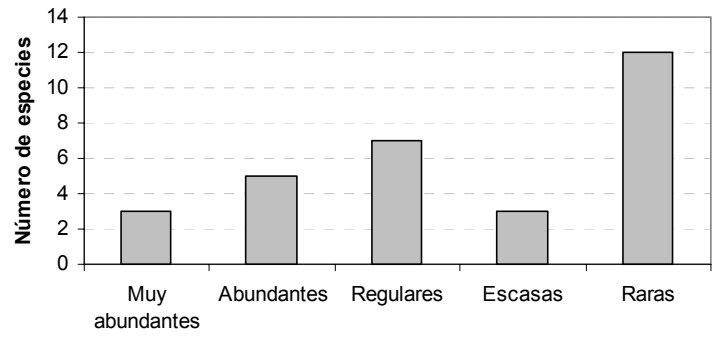

Figura 3. Abundancia de la ictiofauna durante el periodo de estudio

\section{Discusión}

Los resultados de este estudio muestran que durante el periodo post ENSO de los años 1999 y 2000 la ictiofauna de la Bahía Sur presentó diferentes respuestas, así, se pudo observar que la riqueza de especies de peces varía conforme va avanzando el tiempo, encontrándose que, las especies cuya distribución corresponde a la Provincia Panameña, dejan de registrarse dentro de la bahía, mientras que otras especies usuales en la provincia Peruano Chilena se mantienen regularmente en la bahía. Esto se explica por la eventual expansión de la distribución de las especies durante la perturbación, sin embargo conforme pasa el tiempo y las condiciones vuelven a lo usual, las especies también retornan a sus limites de distribución habituales (Hoyos et al., 1985; Hooker, 1998). Estos cambios han sido reportados, sin embargo solo se presentan cuando el ENSO incursiona de manera fuerte en el sistema (Hooker, 1998). Las observaciones también muestran que en una misma bahía especies usualmente alopátricas 
pueden coexistir. Esto nos sugiere que, pasado el punto mas alto de la perturbación (en el periodo entre 1997 y 1998), las respuestas de las especies, como el retorno a sus hábitats usuales, puede demorarse algunos meses mas, posiblemente porque estas pueden generar mecanismos de tolerancia a las condiciones ambientales. Además, este hecho se puede interpretar como la presencia de un período de post ENSO de retorno a las condiciones usuales de la corriente de Humboldt. La coexistencia entre especies sugiere que la competencia es reducida, lo que nos puede llevar a pensar que las especies podrían haber evolucionado bajo las condiciones de variabilidad ocasionadas por los eventos ENSO, tal como lo sugieren Arntz \& Tarazona (1988). Por otro lado, si bien nuestras observaciones no permiten explicar los mecanismos que explican la coexistencia de las especies, se pueden plantear ideas que nos pueden ayudar a comprender este hecho, por ejemplo, durante los eventos ENSO, los peces cambian la composición de sus dietas, alimentándose de lo que el medio les proporciona sin que se vean obligadas a emigrar a nuevos hábitats para encontrar alimento (Hoyos et al., 1985; Sánchez de Benites et al., 1985), así las especies pueden variar sus nichos ante la perturbación y lograr supervivencia.

Los cambios en la riqueza de especies, debido a la paulatina desaparición de los individuos provenientes de la Provincia Panameña así como los datos de distribución (sensu Chirichigno, 1998) de las especies mostrados en este estudio, nos sugieren que en la bahía se llevaron a cabo procesos de resiliencia, con el predominio de las especies habituales para las condiciones no ENSO que caracterizan esta zona, notándose este proceso hacia fines del 2000. Procesos de resiliencia también han sido documentados para comunidades de aves e insectos (Coleoptera), en ecosistemas de lomas adyacentes a litoral (Veliz et al., 2002; Giraldo \& Arellano, 2003).

La variabilidad en la abundancia de las especies, puede ser explicada por los efectos del ENSO, por ejemplo; Se observó grandes cardúmenes de Sphyraena ensis que entraban a la bahía, esta especie es de hábitos pelágico costero, y el desplazamiento de los cardúmenes se debería al acercamiento de las aguas oceánicas, a lugares más someros como la bahía Sur. Para otras especies las abundancias pueden ser explicadas por el tipo de comportamiento, así tenemos; Sphoeroides lobatus es una especie de hábitos solitarios (Goodson, 1988), lo cual explicaría su poca abundancia.

Algunos sesgos deben ser considerados al discutir nuestros resultados, dado que las observaciones se hicieron mediante buceo, esto puede llevar a confusión puesto que, en algunos momentos el agua no presenta las condiciones más adecuadas para la observación, como por ejemplo; turbidez dado la resuspensión de los sedimentos, imposibilitando una correcta identificación de las especies, o dejar sin registrar algún individuo que hubiese estado presente. Sin embargo estudios realizados mediante buceo como el presente, son escasos en nuestro litoral, y su importancia radica en que estos muestran una riqueza de especies relativamente diferente, que no es factible analizar con los métodos usuales de estudios de peces, por lo general mediante artes de pesca.

Como conclusión general, el presente estudio muestra que, durante el post ENSO las especies generan diferentes respuestas, algunas provenientes de la provincia Panameña son capaces de expandir sus límites usuales de distribución hasta la bahía, pero van desapareciendo conforme pasa el tiempo. Otras presentan una gran capacidad de aclimatación ante esta perturbación natural, como las especies que pertenecen a la provincia Peruano - Chilena. Además nuestro estudio muestra la presencia de un período post ENSO, producto del proceso de resiliencia que se produce después de este evento.

\section{Agradecimientos}

Los autores desean agradecer a R. Castillo por su ayuda en revisión del manuscrito.

\section{Literatura citada}

Arntz W. \& Tarazona J. 1988. Una retrospectiva a El Niño 1982-1983: Que hemos aprendido?. En: Salzwedel, H. \& A. Landa (eds.). Recursos y Dinámica del Ecosistema de Afloramiento Peruano. Bol. Inst. Mar Perú-Callao, Vol. Extr. : 353-364.

Chirichigno N. 1998. Clave para identificar los peces marinos del Perú. 2da. Edición, Publicación Especial, Ins. Mar Perú-Callao.

Espino M. 1999. "El Niño 1997-98”: su efecto sobre el ambiente y los recursos pesqueros en el Perú. Rev. Per. Biol. Vol. Extraordinario. : 97-109.

Espino M., Benités C. \& Maldonado M. 1985. Situación de la población de la merluza (Merluccius gayi peruanus) durante "El Niño". En: Arntz, W., A. Landa y J. Tarazona (eds.). "El Niño": Su impacto en la Fauna Marina. Bol. Inst. Mar. Perú-Callao, Vol. Extr. : 159- 162.

Giraldo A. \& Arellano G. 2003. Resiliencia de la comunidad epígea de coleoptera en las lomas de Lachay después del evento el Niño 1997-98. Eco. apl. 2(1): 59-68.

Goodson G. 1988. Fishes of the pacific coast. Stanford University Press. California.

Hoyos L., Tarazona J., Shiga B. \& Chiong V. 1985. Algunos cambios en la ictiofauna y sus relaciones tróficas durante el fenómeno "El Niño" en la Bahía de Ancón. En: Arntz, W., A. Landa y J. Tarazona (eds.). "El Niño": Su impacto en la Fauna Marina. Bol. Inst. Mar. Perú-Callao, Vol. Extr. : 163-171.

Hooker Y. 1998. Fauna asociada a las aguas cálidas presentes en la bahía de Pucusana durante El Niño 
1997-98. Junio a Julio de 1997. Inf. Prog. Inst. Mar Perú. No 85.

Humann P. 1993. Reef Fish identification Galápagos. Ediciones Libri Mundi Quito, Ecuador.

Jahncke J., Checkley D.M. \& Hunt G.L. Jr. 2004. Trends in carbon flux to seabirds in the Peruvian upwelling system: effects of wind and fisheries on population regulation. Fish. Oceanogr. 13: 3, 208223.

Mendo J. \& Wolff M. 2003. El impacto de El Niño sobre la producción de conchas de abanico (Argopecten purpuratus) en Bahía Independencia, Pisco, Perú. Ecol. apl. 2(1): 51-57.

Samame M., Castillo J. \& Mendieta A. 1985. Situación de las pesquerías demersales y los cambios durante el "El Niño". En: Arntz, W., A. Landa y J. Tarazona (eds.). "El Niño": Su impacto en la Fauna Marina. Bol. Inst. Mar. Perú-Callao, Vol. Extr. : 153-158.

Sánchez de Benites G., Alamo A. \& Fuentes H. 1985. Alteraciones en la dieta alimentaria de algunos peces comerciales por efecto del fenómeno "El Niño". En: Arntz, W., A. Landa y J. Tarazona (eds.). "El Niño": Su impacto en la Fauna Marina. Bol. Inst. Mar. Perú-Callao, Vol. Extr. : 135-142.

Velez J. \& Zeballos J. 1985. Ampliación de la distribución de algunos peces e invertebrados durante el fenómeno "El Niño" 1982-1983. En: Arntz, W., A. Landa y J. Tarazona (eds.). "El Niño": Su impacto en la Fauna Marina. Bol. Inst. Mar. Perú-Callao, Vol. Extr. : 173-180.

Veliz C., Sánchez E. \& Tory W. 2002. Cambios espacio temporales en la diversidad de la comunidad de aves de las Lomas de Lachay luego del evento el Niño 97-98. Ecol. apli. (1): 75-79. 


\section{Tabla citada en el texto}

Tabla 1. Lista de las especies encontradas con sus rangos de distribución según Chirichigno (1998). Provincia Peruano - Chilena (P), Provincia Panameña (E), (I.G.= Islas Galápagos).

\begin{tabular}{|c|c|c|c|c|}
\hline Familia & Especie & Nombre vulgar & Distribución & Provincia \\
\hline Bleniidae & Scarthistys gigas & Borracho & Panamá a Chile & $\mathrm{P}$ \\
\hline Bleniidae & Hypsoblennius sordidus & Torito & Caleta la Cruz a Chile & $\mathrm{E}$ \\
\hline Chaetodontidae & Johnrandalia nigrirostris & $\begin{array}{l}\text { Mariposa hocico } \\
\text { negro }\end{array}$ & $\begin{array}{l}\text { Baja California a Cabo } \\
\text { Blanco, Pucusana I.G. }\end{array}$ & $\mathrm{E}$ \\
\hline Chaetodontidae & Chaetodon humeralis & Mariposa común & $\begin{array}{l}\text { Golfo de California a } \\
\text { Pucusana, IG. }\end{array}$ & $\mathrm{P}$ \\
\hline Labridae & Halichoeres dispilus & Doncella & $\begin{array}{l}\text { Golfo de California a b. } \\
\text { Independencia I.G. }\end{array}$ & $P$ \\
\hline Labridae & Bodianus diplotaenia & Vieja colorada & $\begin{array}{l}\text { Baja california a Isla Foca, } \\
\text { Pucusana, Chile }\end{array}$ & $\mathrm{E}$ \\
\hline Pomadasiidae & Anisotremus interruptus & Chita cola amarilla & $\begin{array}{l}\text { Bahía Magdalena a Mancora, } \\
\text { Islas Galápagos }\end{array}$ & $\mathrm{E}$ \\
\hline Pomadasiidae & Anisotremus scapularis & Chita & $\begin{array}{l}\text { Manta (Ecuador) a } \\
\text { Antofagasta, Isla Cocos }\end{array}$ & $\mathrm{P}$ \\
\hline Pomacentridae & Chromis crusma & Castañuela & $\begin{array}{l}\text { Cabo Blanco (Perú) a J. } \\
\text { Fernández (Chile) }\end{array}$ & $\mathrm{E}$ \\
\hline Pomacentridae & Nexilosus latifrons & Castañuela común & $\begin{array}{l}\text { Ecuador a Antofagasta e } \\
\text { Islas Galápagos }\end{array}$ & $\mathrm{P}$ \\
\hline Pomacentridae & Stegastes flavilatus & $\begin{array}{l}\text { Damisela dos } \\
\text { colores }\end{array}$ & $\begin{array}{l}\text { Cabo San lucas hasta Cabo } \\
\text { Blanco, Pucusana }\end{array}$ & $\mathrm{E}$ \\
\hline Pomacentridae & Abudefduf troschellii & Pez Sargento & $\begin{array}{l}\text { Golfo de California a Norte } \\
\text { Perú, Pucusana I.G. }\end{array}$ & $\mathrm{E}$ \\
\hline Serranidae & Mycteroperca xenarcha & Mero negro & $\begin{array}{l}\text { San francisco (EEUU) a } \\
\text { Paita (Perú) }\end{array}$ & $\mathrm{E}$ \\
\hline Serranidae & Ephinephelus labriformis & Mero moteado & B. Magdalena a Paita e I.G. & $\mathrm{E}$ \\
\hline Balistidae & Balistes polylepis & Coche & $\begin{array}{l}\text { Crescent City a Samanco, B. } \\
\text { Independencia }\end{array}$ & $\mathrm{E}$ \\
\hline Belonidae & Strongylura exilis & Pez aguja & $\begin{array}{l}\text { Golfo de California, Isla } \\
\text { chincha, I.G. }\end{array}$ & $\mathrm{E}$ \\
\hline Carangidae & Seriola rivoliana & Fortuno & $\begin{array}{l}\text { Sur de California a norte de } \\
\text { Perú }\end{array}$ & $\mathrm{E}$ \\
\hline Clinidae & Labrisomus phillipii & Trambollo & Paita a Coquimbo (Chile) & $\mathrm{P}$ \\
\hline Cheilodactylidae & Cheilodactylus variegatus & Pintadilla & $\begin{array}{l}\text { Paita (Perú) a Talcahuano } \\
\text { (Chile) }\end{array}$ & $\mathrm{P}$ \\
\hline Fistularidae & Fistularia corneta & Pez corneta & $\begin{array}{l}\text { Golfo de California a Paita, } \\
\text { Callao }\end{array}$ & $\mathrm{E}$ \\
\hline Khyposidae & Doidixodon laevifrons & Babunco & Isla Huañape a Tatal & $\mathrm{P}$ \\
\hline Mugilidae & Mugil cephalus & Lisa & B. San Franciscos a Valdivia & $\mathrm{P}$ \\
\hline Mullidae & Pseudopeneus grandisquamis & Salmonete & Baja California a Chile & $\mathrm{P}$ \\
\hline Oplegnathidae & Oplegnathus insignis & Loro & $\begin{array}{l}\text { Pto. Pizarro a Antofagasta } \\
\text { I.G. }\end{array}$ & $\mathrm{P}$ \\
\hline Sparidae & Calamos brachypomus & Marotilla & $\begin{array}{l}\text { California a I. Lobos de } \\
\text { tierra, La Punta }\end{array}$ & $\mathrm{P}$ \\
\hline Scaridae & Nicholsina denticulada & Pococho & $\begin{array}{l}\text { Golfo de California a Islas } \\
\text { Chincha, I.G. }\end{array}$ & $\mathrm{E}$ \\
\hline Scorpaenidae & Scorpaena histrio & Pez diablo & $\begin{array}{l}\text { Cabo San Lucas a Pucusana, } \\
\text { I.G. }\end{array}$ & $\mathrm{P}$ \\
\hline Scombridae & Scomberomorus sierra & Sierra & $\begin{array}{l}\text { Sur de California a Paita } \\
\text { (Perú) }\end{array}$ & $\mathrm{E}$ \\
\hline Sphyraenidae & Sphyraena ensis & Barracuda & $\begin{array}{l}\text { Golfo de California a Lobos } \\
\text { de afuera, Ancón }\end{array}$ & $\mathrm{E}$ \\
\hline Tetraodontidae & Sphoeroides lobatus & Tamborín & $\begin{array}{l}\text { Redondo beach a Paita, Isla } \\
\text { Don Maritin, I.G. }\end{array}$ & $\mathrm{P}$ \\
\hline
\end{tabular}

\footnotetext{
${ }^{1}$ Rainforest Expeditions. Av. Aramburu 166 Dpto. 4B, Lima 18. Email: balanusperu@yahoo.com.mx

${ }^{2}$ Laboratorio de Biología y Ecología Marina, Universidad Nacional Agraria La Molina. Email:

babuchapv@yahoo.com
} 